\title{
The Need to Implement Modelling and Simulation as Part of the Curriculum in Basic Engineering Courses
}

\author{
Mohammed Ghazy \\ Dept. of Aerospace Engineering, King Fahd University of Petroleum \& Minerals \\ Dhahran 31261, Saudi Arabia \\ Dept. of Engineering Math \& Physics, Alexandria University \\ Alexandria 21544, Egypt \\ Tel: 966-13-860-3769Ｅ-mail: mohammed.ghazy@kfupm.edu.sa
}

Atef Ata

Dept. of Engineering Math \& Physics, Alexandria University

Alexandria 21544, Egypt

Tel: 20-106-282-6550Ｅ-mail: atefa@alexu.edu.eg

Received: April 11, 2020

Accepted: June 23, 2020

Published: July 23, 2020

doi:10.5296/gjes.v6i2.16828

URL: https://doi.org/10.5296/gjes.v6i2.16828

\begin{abstract}
In this paper a proposal for implementing modelling and simulation techniques into the curriculum of basic engineering courses is introduced. Justifications and case studies from some engineering courses are discussed. Examples from particle and rigid body motions in engineering mechanics course and other related courses are emphasized. In this proposal we introduce a method of simplification of basic concepts in applied engineering sciences. The concept of course project can be utilized to encourage students to apply their basic knowledge on modelling and simulation of some basic topics such as projectile and constrained motions. This proposal has the advantages of communicating concepts in science through the daily use of communication technology among students and instructors. Simulation can attract other students, who find difficulty understanding engineering problems, to the engineering profession. In addition, the new proposal can be applied with no additional cost on the institution, no extra credit hours for students, and a minimum additional load on the instructor.
\end{abstract}

Keywords: Modelling and simulation, Curriculum, Engineering education 


\section{Introduction}

The motion of particles and rigid bodies are usually explained to students in basic engineering courses. Sometimes, students need to use their imagination to understand these motions. If some students can't understand the basic concepts, it will be a problem in all related courses through their degree. This problem will be significant when the basic courses are pre-requisites for higher courses. The use of nowadays common and simple modelling and simulation software and tools makes delivering such concepts easier. Currently, computer simulation programs are used mainly in advanced senior undergraduate courses or postgraduate courses. With the current progress in using technology applications in our daily life and for education purposes, even fresh engineering students can use these programs with no extra efforts or credit hours to help them understanding some concepts. Engineering courses that require more visualization may need simulation programs to be built inside the curricula. In the undergraduate curricula, examples for such courses are; engineering mechanics, engineering physics, as basic courses, and theory of machines and mechanisms as advanced courses. While in the postgraduate curricula, courses such as flight dynamics and control, and orbital mechanics can get benefits from using simulation.

Modelling and simulation in engineering discipline have many definitions. One of the most suitable for engineering refers to a combination of processes in which a system's behavior is demonstrated or predicted by a reductive computational representation (Magna, 2017). Within education contexts, modelling and simulation skills and tools can be used to further support the integration of both divergent and convergent thinking (Dym et. al., 2005). Therefore, modelling and simulation skills are integrated across many engineering and science disciplines as analytic tools that facilitate the study of complex phenomena and as a predictive tool to anticipate the suitability of new designs (Magna, 2017).

Engineering students, usually, study most of the particle or rigid body motion related problems in one or two dimensions. The problems that deal with representation of the motion in three-dimensional space are few. Moreover, discussing the three-dimensional problems by representation on the two-dimensional sheet of paper is, sometimes, not effective for the students to understand the motion. Implementing the electronic space in teaching would solve this problem and give visualization ability that normal teaching styles lack.

It is a fact that computer tools become more dependable in teaching and grading engineering courses. When grading for large number of students, it is natural to implement them extensively in the learning process (Ghazy \& Ata, 2019; 2020). Some research efforts are conducted to build visualization tools to help students studying engineering mechanics (Constans et al., 2002). Even, some recent engineering text books take into consideration the new attitudes in learning methodology such as flipped classroom and other self-learning tools (Daqaq, 2018).

The concept of course projects at the end of each undergraduate or postgraduate course can motivate students to apply their basic knowledge in modelling and simulation. There are many basic engineering problems that can be modelled using basic laws in the student's textbook and simulation of these problems makes studying these courses an interesting 
process. Examples of these problems include projectile motion in a non-resistive and a resistive media, constrained motion for the roller coaster, and some basic mechanisms in theory of machines. More specifically, students can use the basic equations in their textbooks and introduce some variables such as time and space to simulate the time history of any point in the mechanism or the motion of the projectiles. It is noteworthy that some textbooks introduce this concept of modelling and simulation through computer-based problems at the end of each chapter to motivate the creativity of students and present visual tool to satisfy their imagination. However, in most cases this part is considered as supplementary material and not essential in the course syllabus. Nowadays, some big publishers offer online learning facilities to help students exercise and instructors to give online quizzes and managing class grades and attendance. However, publishers usually offer these facilities when students and instructors buy their textbooks (Daqaq, 2018; Hibbeler, 2013; Meriam, 2018). As a result, these facilities, despite their enormous capabilities, can't be accessed by students and instructors in many countries.

In this paper we introduce, analyze, and justify a proposal to modify the curricula of some basic engineering courses to implement modelling and simulation as basic content. In section II, an exploratory analysis is given. Two classical engineering dynamics problems about motion of particles are solved and the motion is represented graphically, and methods of simulation and extra visualization are proposed. In section three, we discuss the findings and conclusions are drawn.

\section{Case Studies}

\subsection{Example (1) Rectilinear Motion of a Particle}

The rectilinear motion of a particle is described by the following equation of the acceleration,

$$
a=3 t(t-4)+11
$$

where $t$ is the time in seconds and $\mathrm{a}$ is the acceleration in $\mathrm{m} / \mathrm{s}^{2}$. Using the initial conditions $s(0)=0, \frac{d s}{d t}(0)=-6(m)$ of the position and velocity respectively and integrating (1) twice gives the position along the $x$ axis as a function of time

$$
s(t)=\frac{1}{4} t^{4}-2 t^{3}+\frac{11}{2} t^{2}-6 t
$$

To understand the motion, the student has to calculate the positions at which the particle will stop temporarily and reverse its motion. Particle will stop at the moments $t=1 \mathrm{sec}, t=2 \mathrm{sec}$, and $t=3 \mathrm{sec}$, respectively. The corresponding positions to these moments are $s(1)=-2.25(m), s(2)=-2(m)$, and $s(3)=-2.25(m)$.

Figure (1) depicts the distance segments on the straight line from start, between the points of temporary stops, and till the particle passes by its initial position again after four seconds. 


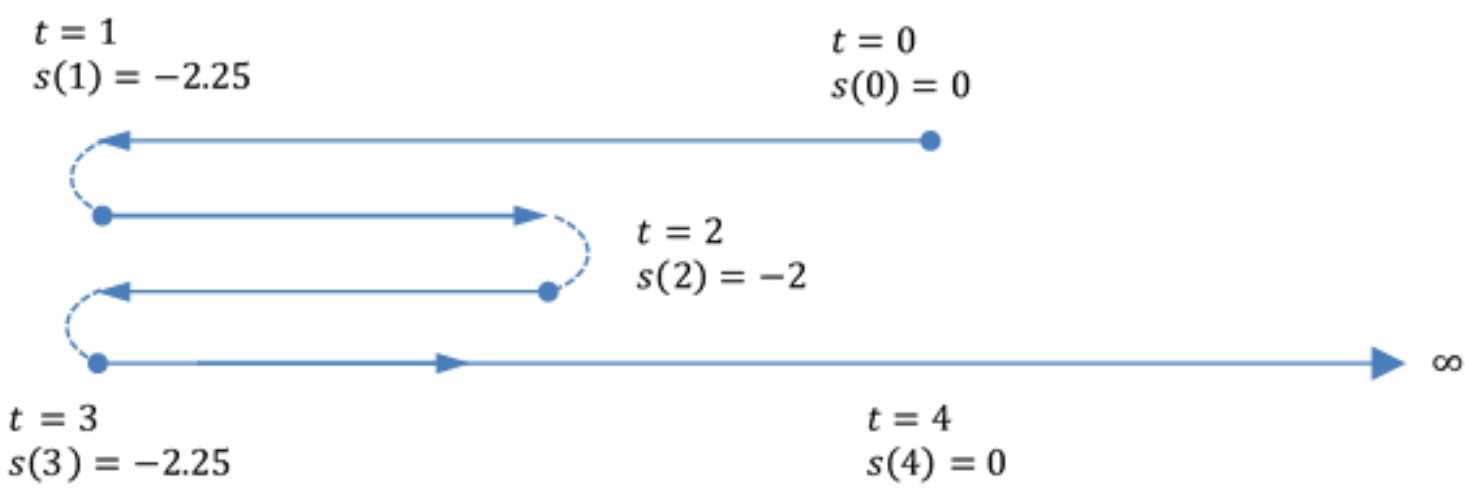

Figure 1. Details of Rectilinear motion of a particle (not to scale)

In figure 2, the position of the particle is plotted with time. Though it is important, it doesn't give much more useful information, from the point of view of visualization, than figure 1. Even with graphical representation, a student may think that the motion is curvilinear not rectilinear because of the vertical shift between line segments. Therefore, we better make a simulation in which a particle moves back and forth on the same line. This simulation will be more useful and doesn't lead to any conflict.

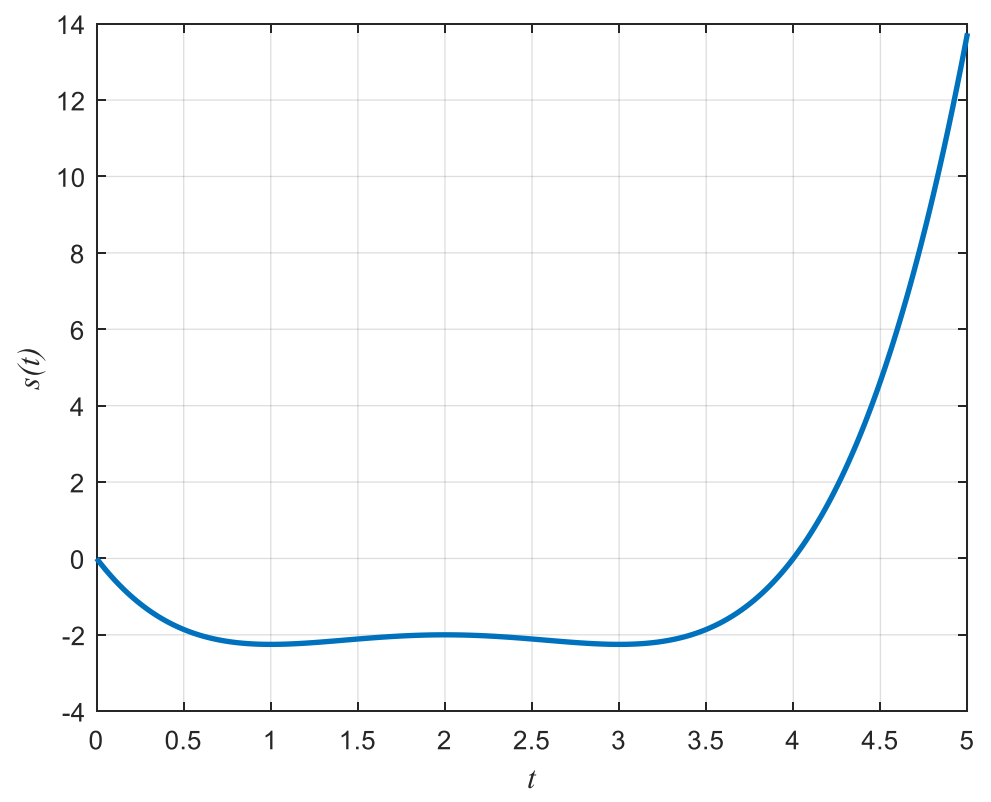

Figure 2. Particle's Position with time

An important thing that can be represented graphically is the speed of the particle. As indicated in figure 3, a student will be able to know the velocity value and sign at any time in addition to its initial and final values. He can also realize when it increases or decreases, and when it will be a minimum or a maximum. 


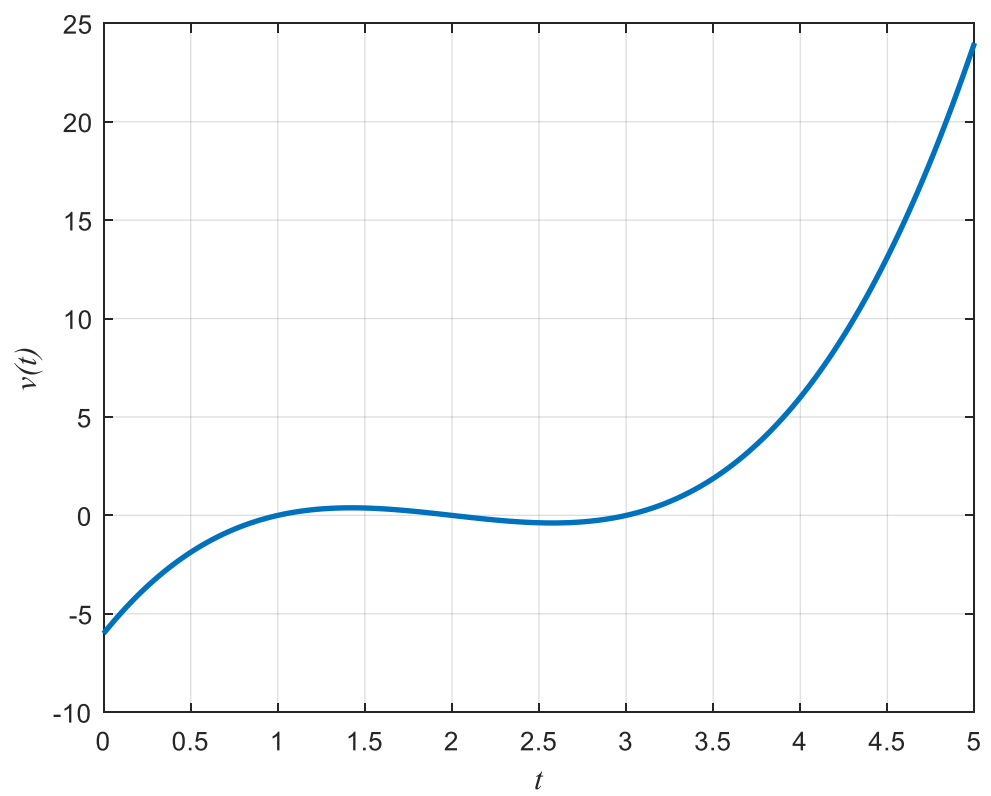

Figure 3. Velocity of the particle with time

linking position and speed at the same moment requires more efforts. Realizing the position, velocity, and acceleration at the same moment even requires more efforts. In figure 4, the velocity of the particle is plotted with its position. Without tracking the motion point by point it will be difficult understanding the behavior of the particle especially along the closed loop between $s=-2$ and $s=-2.25$.

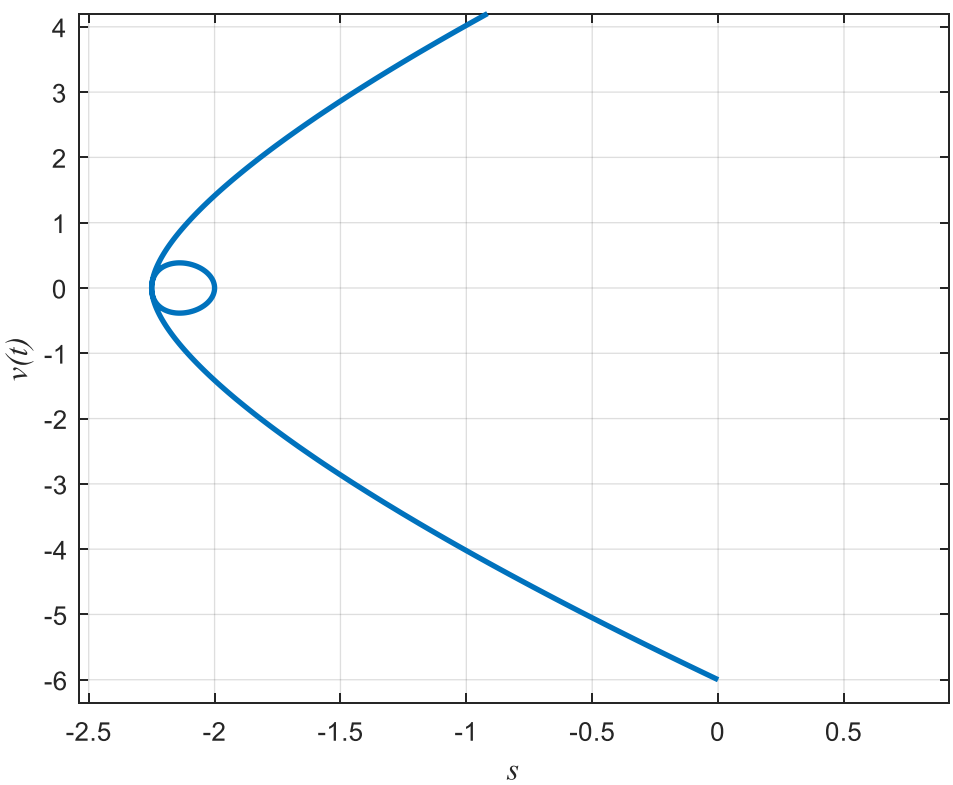

Figure 4. Velocity of the particle with its position 


\section{Macrothink}

\subsection{Example (2): Nonlinear Pendulum Model}

In this example a student should understand and analyze the two-dimensional motion of a simple pendulum in addition to finding the equation of motion of its mass. The objective of this example is to investigate the performance of the pendulum shown in Figure 5. This is a good opportunity for undergraduate students to understand the dynamic behavior of a pendulum that can be simulated as a machine or a robot. The considered pendulum consists of a massless rod connected to a frictionless pivot at one end and attached to a concentrated mass $m$ at its free end.

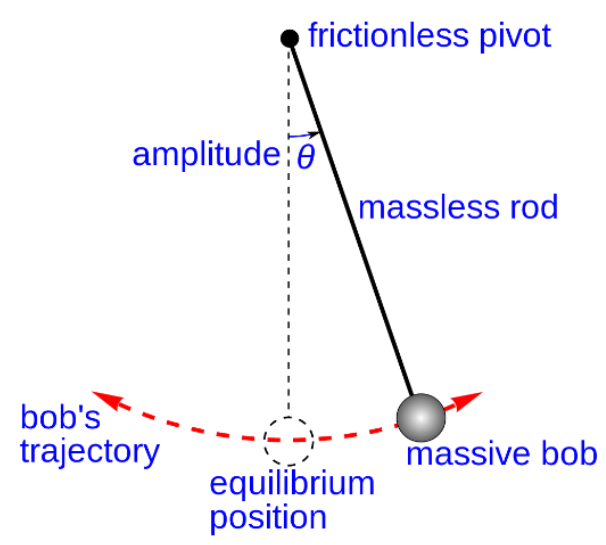

Figure 5. Vertical simple pendulum

In figure 5, the length of the rod is $L$ and the angle of the rod with the vertical line $\theta$ is the dependent variable. In case no other external forces exist except the weight of mass, the equation of motion in terms of $\theta$ reads

$$
\ddot{\theta}+\frac{g}{L} \sin \theta=0
$$

Knowing that $g=9.81\left(\mathrm{~m} / \mathrm{s}^{2}\right)$ and $L=1(\mathrm{~m})$, a student is required to plot $\theta$ with time using two cases of initial conditions of the angle; $\theta(0)=0.5(\mathrm{rad})$ and $\theta(0)=0.8 \pi(\mathrm{rad})$. For these two cases, the initial angular velocity is assumed to be $\dot{\theta}(0)=0\left(\frac{\mathrm{rad}}{\mathrm{s}}\right)$. The student should be able to investigate how linearization can alter the solution slightly and how to check the accuracy of the simulation outputs.

Usually in basic engineering courses, the students use the small angle approximation $\sin \theta \approx \theta$. When applying this approximation to (3) it reduces to

$$
\ddot{\theta}+\frac{g}{L} \theta=0
$$

Equation (4) is a linear equation and its solution can be determined in a closed form as: 


$$
\theta(t)=\theta(0) \cos \sqrt{\frac{g}{L}} t
$$

Equation (5) represents a pure oscillation of amplitude $\theta(0)$ and time period $T_{P}=\frac{2 \pi}{\sqrt{g / L}}=2(s)$, which doesn't depend on the initial conditions. This time period and the initial conditions will be used to select the simulation final time to facilitate comparing the approximate solution to the exact solution. Figure 6 shows the angle $\theta$ with time using the solution in (5).

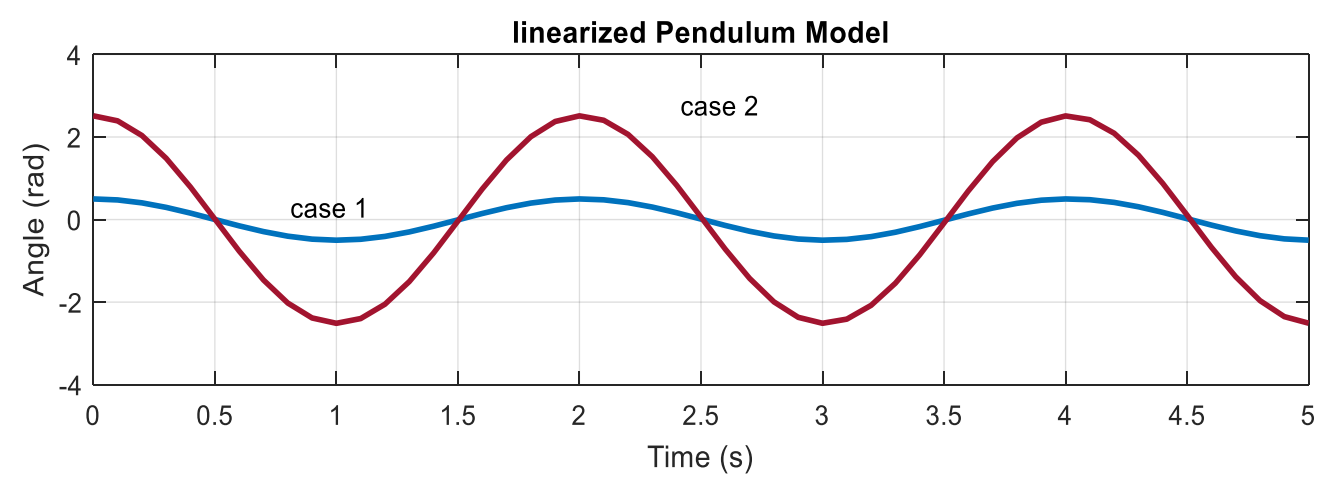

Figure 6. Effect of changing the initial conditions on the linearized pendulum model

In this problem, when we relax the assumption of a small angle, the linearized solution doesn't approximate very well the original nonlinear system in (3). As the analytical solution of the nonlinear problem requires advanced mathematical knowledge that would be difficult for fresh or sophomore students, a numerical integration using available software such as MATLAB could be useful. First, the problem should be formulated into a state-space representation where the states are $\left\{x_{1}, x_{2}\right\}=\{\theta, \dot{\theta}\}$, and the state equations will be

$$
\begin{gathered}
\dot{x}_{1}=x_{2} \\
\dot{x}_{2}=-\frac{g}{L} \sin x_{1}
\end{gathered}
$$

The results are shown in Figure 7, where $\theta$ is plotted with time for five seconds.

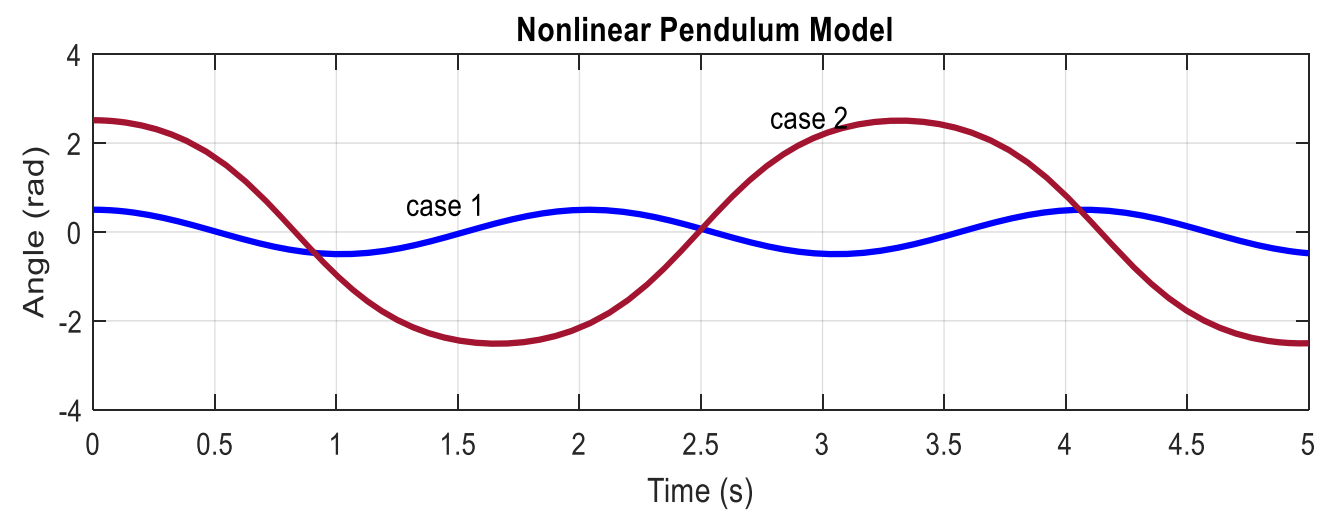

Figure 7. Effect of changing the initial conditions on the nonlinear pendulum model 
By simulating the history of the pendulum angle with time, students can clearly observe the effect of the nonlinearity in the pendulum model and how linearization can affect the solution slightly. Also, they can realize from the two Figures how the initial conditions change the solution dramatically. It is obvious, from Figure 7, that the period of oscillation when initial angle is $\theta(0)=0.5$ (rad) is not the same as when it is $\theta(0)=0.8 \pi(\mathrm{rad})$. In figure 7 , one can notice that the larger the initial angle $\theta(0)$, the larger the period of oscillation. In case of $\theta(0)=0.8 \pi(\mathrm{rad})$, the period is $3.25 \mathrm{sec}$ and the relative percent error if we use the linearized model will be $41.67 \%$.

\section{Conclusion}

This research indicated that including modelling and simulation in basic engineering courses is necessary. It is clear from the case studies and discussion that introducing modelling and simulation topics in undergraduate courses enhances the students' understanding. It helps students to build their confidence in the subjects they studied and passed in basic engineering sciences. This research indicated that, students can apply, with no extra cost, their basic knowledge in modelling such as Newton's second law of motion and basic simulation software to enhance their perception to many phenomena in engineering subjects.

A very important issue in engineering education which is nonlinearity is highlighted in our work. It is shown that linearization is not enough and nonlinear phenomenon is better understood by a student when implementing modelling and simulation.

The course project at the end of each semester is an excellent opportunity for both students and instructors to apply modelling and simulation. Students can apply and integrate their basic knowledge with some computer skills to understand the behavior of simple and complex systems. For instructors, some simulation results and projects can be presented in related conferences and journals after some modifications to allow students to participate and encourage them to be more creative. It is an opportunity also to enhance the competitions among students when they present their valuable ideas.

The future work of this research includes proposing a questionnaire to get feedback from students as well as the instructors about the usefulness of this approach in promoting engineering understanding of the basic subjects.

\section{References}

Alejandra, J. M. (2017). Modelling and Simulation in Engineering Education: A learning Progression. Journal of Professional Issues in Engineering Education and Practice, 143(4), 1-19. https://doi.org/10.1061/(ASCE)EI.1943-5541.0000338

Constans, E., Kadlowec, J., Sukumaran, B., von Lockette, P., \& Cleary, D. (2002). Hands-on Learning Tools for Engineering Mechanics. Proceedings of the 2002 American Society for Engineering Education, Annual Conference \& Exposition, Montreal, Canada. https://peer.asee.org/10616.

Daqaq, M. F. (2018). Dynamics of Particles and Rigid Bodies: A Self-Learning Approach. Wiley-ASME Press Series. https://doi.org/10.1002/9781119463207 


\section{Macrothink}

Global Journal of Educational Studies

ISSN 2377-3936 2020, Vol. 6, No. 2

Dym, C. L., Agogino, A. M., Eris, O., Frey, D. D., \& Leifer, L. J. (2005). Engineering design thinking, teaching, and learning. Journal of Engineering Education, 94(1), 103-120. https://doi.org/10.1002/j.2168-9830.2005.tb00832.x

Ghazy, M., \& Ata, A. (2019). Computer-Based Examinations in Engineering Mechanics Course; Necessity and Benefits. Proceedings of the Frist International Conference on Pedagogical Innovation and Technology Enhanced Learning, 26-27.

Ghazy, M., \& Ata, A. (2020). MCQ Examinations for Large Number of Students: Design and Grading. International Journal of Education, 12(01), 118-126. https://doi.org/10.5296/ije.v12i1.16319

Hibbeler, R. C., \& Yap, K. B. (2013). Mechanics for Engineers: Dynamics (SI Edition, 13/E). Prentice Hall.

Meriam, J. L., Kraige, L. G., \& Bolton, J. N. (2018). Engineering Mechanics: Dynamics (9th ed.). John Wiley \& Sons, Inc.

\section{Copyright Disclaimer}

Copyright for this article is retained by the author(s), with first publication rights granted to the journal.

This is an open-access article distributed under the terms and conditions of the Creative Commons Attribution license (http://creativecommons.org/licenses/by/3.0/). 